\title{
Integrated Optical Frequency Shifter on a Silicon Platform
}

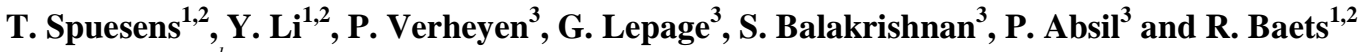 \\ ${ }^{1}$ Photonics Research Group, INTEC, Ghent University - imec, 9000 Gent, Belgium \\ ${ }^{2}$ Center for Nano- and Biophotonics, Ghent University, 9000 Ghent, Belgium \\ ${ }^{3}$ imec, Kapeldreef 75, 3001 Leuven, Belgium \\ thijs.spuesens@intec.ugent.be
}

\begin{abstract}
An integrated optical frequency shifter on a standard silicon photonics platform completely fabricated in a CMOS pilot line and showing $\mathrm{MHz}$ frequency shifts with a sideband suppression ratio up to $36 \mathrm{~dB}$ is demonstrated.

OCIS codes: (130.4110) Modulators; (130.7405) Wavelength conversion devices; (130.6622) Subsystem integration and techniques
\end{abstract}

\section{Introduction}

Optical frequency shifting (OFS) is a technique that is widely used in systems that rely on heterodyne interferometry such as e.g. Laser Doppler Vibrometry (LDV) [1], but is also considered for advanced optical modulation formats that offer high spectral efficiency such as orthogonal frequency division multiplexing (OFDM) [2].

In currently available commercial systems acousto-optic modulators (AOMs) are used as optical frequency shifters and offer high conversion efficiency (CE), indicating the additional loss induced by the frequency shifting process, as well as high sideband suppression ratio (SSR). However, AOMs typically suffer from limited tuning range and bandwidth. Another major drawback is that the working principle of AOMs is not easily implemented in integrated planar waveguide circuits and prevents these applications to benefit from the numerous advantages of integration. Several alternatives have been proposed to realize integrated frequency shifters $[3,4]$. An OFS on a silicon platform showing good SSR has been demonstrated using a serrodyne approach, but the limited bandwidth of the thermo-optic phase shifters used, makes it impossible to reach frequency shifts in the desired MHz range [3]. Carrier depletion based silicon modulators can offer higher bandwidth, but are known to suffer from spurious amplitude modulation, thereby decreasing the SSR. An alternative to the serrodyne approach is to use a 4-branch Mach-Zehnder modulator (MZM), but there it is in general difficult to realize a high SSR and CE at the same time. Good performance of such an OFS has been demonstrated on a silicon organic hybrid (SOH) platform by shaping the drive signal but, while it is a CMOS compatible technology, the polymers tend to suffer from stability issues [4].

Here we propose a variation on the serrodyne implementation, similar to a fiber based demonstration [5], whereby the linear phase ramp to be realized is divided over two phase modulators. In this way, the reset of the phase modulators will have almost no impact on the output signal, thereby allowing for a tremendous simplification of the drive signal. Furthermore, because the maximum required phase excursion of the phase modulators is now limited to $\pi$ instead $2 \pi$ compared to the conventional serrodyne approach, the impact of spurious amplitude modulation is significantly reduced.

\section{Principle}

The working principle of our proposed frequency shifter is schematically illustrated in Figure 1(a). Light enters a $1 \times 2$ switch, which is driven by a block signal with a frequency equal to the desired frequency shift. The first half of the switching period the input light is directed towards phase modulator 1 (PM1), while the second half the input light is directed towards phase modulator 2 (PM2). The phase modulators (PMs) are driven in push-pull by a signal that contains a linearly increasing slope, such that the phase shift is ramped up from 0 to $\pi$ in half the switching period. PM2 is followed by a static phase shifter that induces a $\pi$ shift. The outputs of both modulators are recombined either by a switch or a $3 \mathrm{~dB}$ combiner. After recombining, a linearly increasing slope from 0 to $2 \pi$ over the full switching period is obtained at the output, hence resulting in the desired frequency shift.

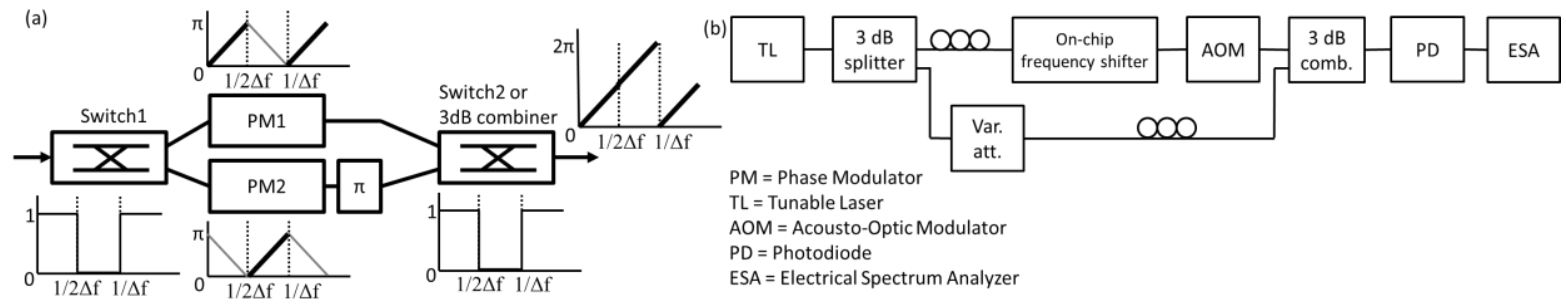

Figure 1: (a) Schematic overview showing the working principle of the proposed frequency shifter. (b) Schematic representation of the measurement setup. 


\section{Experimental results}

Samples with the proposed frequency shifter were fabricated in imec's iSiPP25G platform [5]. The input switch is implemented as a balanced MZI configuration with PMs in both arms, while the output switch was replaced by a 3 $\mathrm{dB}$ combiner to lower the complexity for this proof of concept. The 2 PMs in the switch, PM1 and PM2, are all implemented as $2 \mathrm{~mm}$ long carrier depletion silicon phase modulators. DC characterizations of these phase modulators showed an insertion loss of $1.5 \mathrm{~dB} / \mathrm{mm}$ and a $\mathrm{V} \pi \mathrm{L} \pi$ of $1.8 \mathrm{Vcm}$. The total insertion loss of the frequency shifter is measured to be $9.4 \mathrm{~dB}$, which can be broken down to $3 \mathrm{~dB}$ from the switch, $3 \mathrm{~dB}$ from the PMs, $3 \mathrm{~dB}$ from the passive combiner and $0.4 \mathrm{~dB}$ excess loss from other passives. However, in future designs the loss can be reduced by e.g. replacing the $3 \mathrm{~dB}$ combiner by a switch and using shorter PMs for these switches. Grating couplers with a peak efficiency of $-3 \mathrm{~dB}$ at a wavelength of $1560 \mathrm{~nm}$ were used to couple light in and out of the chip.

The measurement setup is schematically shown in Figure 1(b). The output of a tunable laser was split using a 3 $\mathrm{dB}$ coupler where one arm goes to the silicon PIC containing the optical frequency shifter and subsequently an external AOM, while the other arm is used as reference. Both arms are then recombined in another $3 \mathrm{~dB}$ coupler and connected to a photodiode. The output of the photodiode is connected to an electrical spectrum analyzer. The external AOM, inducing a frequency shift of $200 \mathrm{MHz}$, is included to be able to measure the conversion efficiency of the integrated frequency shifter. Arbitrary Waveform Generators (AWGs), which were locked in frequency and phase, were used to generate the block signal for the switch and the triangular or sinusoidal signals for the PMs. Both for the switch and for the PMs a dual-drive push-pull modulation scheme was used, but a single-drive scheme can also be applied. In a first measurement we applied a block signal of $1 \mathrm{MHz}$ to the switch, while a triangular signal of $1 \mathrm{MHz}$ was applied to the two PMs. Optimum performance was obtained when the switch modulators were driven by a voltage swing of $4.3 \mathrm{Vpp}$ and biased at 2.5 and $2.7 \mathrm{Vdc}$, while PM 1 and 2 required a voltage swing of 9 Vpp with a bias of 4.7 and $4.5 \mathrm{Vdc}$, respectively. The electrical spectrum shown in Figure 2(a) reveals indeed a 1 MHz frequency shift with respect to the $200 \mathrm{MHz}$ shift induced by the external AOM. An SSR of $36 \mathrm{~dB}$ is obtained, while the $\mathrm{CE}$ is as low as $0.2 \mathrm{~dB}$. In a second measurement we aimed for a larger frequency shift and used a $20 \mathrm{MHz}$ block signal for the switch, while for the PMs we used a $20 \mathrm{MHz}$ sinusoidal drive signal of $7.8 \mathrm{Vpp}$ because the AWG used was not able to generate triangles with a frequency of $20 \mathrm{MHz}$. However, despite the reduced linearity of the phase ramp when using a sinusoidal drive signal, we still obtained an SSR of $27.3 \mathrm{~dB}$ and a CE of $2.2 \mathrm{~dB}$ as can be seen in Figure 2(b). Higher frequency shifts should also be feasible as the small-signal bandwidth for these modulators was measured to be $\sim 4 \mathrm{GHz}$ at $0 \mathrm{~V}$ bias.
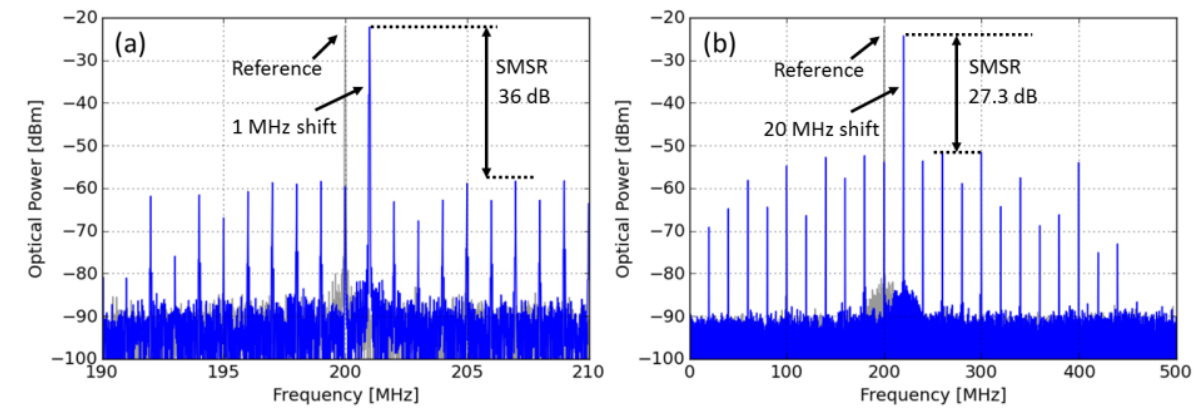

Figure 2: Measured electrical spectra with (blue) and without (grey) additional frequency shift in case of (a) a $1 \mathrm{MHz}$ shift with a triangular drive signal and (b) a $20 \mathrm{MHz}$ shift with a sinusoidal drive signal.

\section{Conclusion}

In this paper, we demonstrated an integrated optical frequency shifter with an SSR up to $36 \mathrm{~dB}$ and a CE of only 0.2 $\mathrm{dB}$. This is, to the best of our knowledge, the first demonstration of such a device being fully fabricated in a CMOS pilot line and has the potential to realize $\mathrm{GHz}$ frequency shifts with large spectral purity and efficiency. Small modifications to reduce the $9.4 \mathrm{~dB}$ insertion loss in this proof of concept have been presented. This demonstration makes it possible to realize a fully integrated heterodyne LDV system on a standard silicon photonics platform.

Acknowledgment: Funding is acknowledged from EU FP7 under grant agreement No 318178 PLAT4M.

[1] P. Castellini et al., "Laser Doppler Vibrometry: Development of advanced solutions answering to technology's needs". Mechanical Systems and Signal Processing, 20(6), 1265-1285, (2006).

[2] Y. Ma et al., "1-Tb/s single-channel coherent optical OFDM transmission over 600-km SSMF fiber with subwavelength bandwidth access". Opt Express, 17(11), 9421-9427, (2009).

[3] Y. Li et al., "Optical frequency shifter on SOI using thermo-optic serrodyne modulation." GFP, p.75-77 (2010).

[4] M. Lauermann et al., "Integrated silicon-organic hybrid (SOH) frequency shifter." OFC, p. Tu2A-1, (2014).

[5] C.S. McDermitt et al., "RF frequency shifting via optically switched dual-channel PZT fiber stretchers," in Microwave Theory and Techniques, IEEE Tran. on , 53(12), pp.3782-3787, 2005

[6] P. Absil et al., "imec iSiPP25G silicon photonics: a robust CMOS-based photonics technology platform" SPIE, p. 93670V, (2015). 